\title{
Bandwidth Control of Second Harmonic Generation through Chirped Period Poling of Optical Fibres
}

\author{
Albert Canagasabey, Costantino Corbari, Peter G. Kazansky, Morten Ibsen \\ Optoelectronics Research Centre, University of Southampton, SO17 1BJ, United Kingdom
}

Precise control of the bandwidth of second harmonic generation in silica fibres is realised through chirped period poling. The control of the acceptance bandwidth allows the frequency doubling of ultra-short pulsed laser sources.

The centrosymmetric nature of amorphous silica can be broken through thermal poling. Thermal poling is a technique through which the application of heat and an electric field can relocate ions, causing an electric field to be built up within the glass matrix which is "frozen-in" once the heat is removed. The frozen-in field combines with the inherent third order nonlinearity $\left(\chi^{(3)}\right)$ of the glass to give the effective second order nonlinearity $\left(\mathrm{SON}, \chi^{(2)}\right)$ :

$$
\chi^{(2)}=3 \chi^{(3)} E_{d c}
$$

This induced $\chi^{(2)}$, although relatively small, can be utilised to fabricate devices based on the SON, such as, electrooptic modulators, optical switches, frequency converters, optical parametric oscillators, etc. When compared to nonlinear crystals for frequency doubling, thermal poling of silica fibres allows longer interaction lengths which compensates for the lower nonlinearity, lower losses and straightforward integration into all-fibre systems. Second harmonic generation (SHG) has been demonstrated in quasi phase matched (QPM) silica fibres with a conversion efficiency exceeding $20 \%$ realised through the use of photo-lithographically defined electrodes [1]. More recently SHG using periodic ultra-violet (UV) erasure of a uniformly poled twin-hole fibre was demonstrated [2]. Point-bypoint periodic UV erasure immensely simplifies the construction process of the QPM structure; it is compatible with the fabrication of metre-long periodically poled fibre devices and allows greater flexibility in the engineering of the period while maintaining tight QPM tolerances. In QPM structures, the conversion efficiency $(\eta)$ grows proportional to the square of the interaction length $(L)$ and the fundamental power $\left(P_{\omega}\right)$ while the bandwidth $\left(\lambda_{B W}\right)$ of the generated second harmonic (SH) scales inversely with the interaction length:

$$
\eta \propto P_{\omega}^{2} L^{2} \quad(2) \quad \lambda_{B W} \propto 1 / L
$$

The optical bandwidth of grating components such as fibre Bragg gratings (FBG) can be broadened by introducing a chirp into the grating structure. This technique can also be adapted to the case of QPM-SHG to modify the acceptance bandwidth of the QPM process to overcome the inevitable narrowing of the bandwidth for longer devices. In fact this technique has been successfully implemented in crystals such as $\mathrm{LiNbO}_{3}$ and $\mathrm{KTiOPO}_{4}$ for frequency doubling of femtosecond sources and pulse compression [3]. Theoretical predictions of such chirped structures indicate that the QPM acceptance bandwidth can be broadened without significant loss to the conversion efficiency for extended interaction lengths [4]. Thus the acceptance bandwidth can be increased to accommodate broadband ultra-short mode-locked high power sources for frequency conversion. In this work, the broadening of the QPM acceptance bandwidth through chirped UV erasure is experimentally demonstrated in silica fibres for the first time.

Silica fibre of numerical aperture 0.20 was fabricated with two holes on either side of the core to accommodate electrodes for the application of the electric field. One of the holes was intentionally positioned closer to the core to attain better overlap with the induced nonlinear layer which is formed around the anode electrode hole during the thermal poling process. For the purpose of in-situ measurement of the $\chi^{(2)}$ during poling, the electrodes are inserted into polished holes in the sides of the fibre. The twin-hole fibre device is then fusion spliced into one arm of an allfibre Mach-Zehnder interferometer. Thus the growth of the induced SON is monitored by the online measurement of the electro-optic coefficient through the change in phase in the fibre device during thermal poling [5]. A SON value of $\sim 0.15 \mathrm{pm} / \mathrm{V}$ was measured for the fibre used for this experiment.

For SHG, the uniformly poled fibres were periodically erased over a length of $8 \mathrm{~cm}$ using $244 \mathrm{~nm}$ photons from a continuous-wave frequency doubled argon ion laser. Poled fibres were also erased with a linearly varying period 
(chirp) for the purpose of broadening the acceptance bandwidth. The centre period $(\Lambda)$ was maintained at $\sim 52.85$ $\mu \mathrm{m}$ for the devices. A wavelength tunable (1535-1562 nm) master oscillator power amplifier (MOPA) laser source of narrow linewidth $(\sim 15 \mathrm{pm}), 10 \mathrm{~ns}$ pulses duration, $450 \mathrm{kHz}$ repetition rate was employed to characterise the QPM devices [2]. The average power coupled into the poled fibre device was $140 \mathrm{~mW}$.
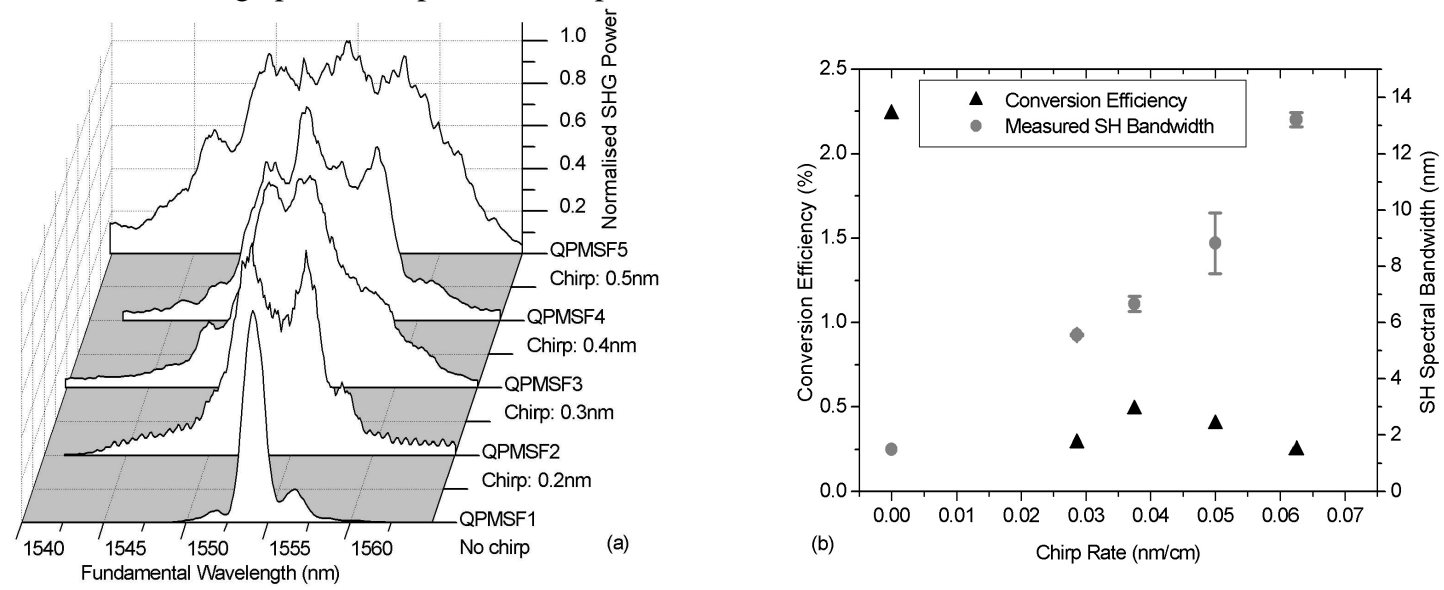

Figure 1(a): SHG tuning curves for quasi phase matched silica fibre (QPMSF) of varying chirp shown as a function of the fundamental wavelength. (b): the dependence of SH conversion efficiency and SH spectral bandwidth on the chirp rate of the QPM structure. The bars show the deviation from the expected values.

Figure 1(a) shows the wavelength tuning curves of the generated second harmonic for five QPM devices of identical lengths, one with no chirp and four with linearly varying chirps $(\Delta \Lambda)$ of $0.2,0.3,0.4$ and $0.5 \mathrm{~nm}$. The profiles of the tuning curves for the chirped devices are in good agreement with theoretical predictions of chirped waveguides [4]. The bandwidths of the chirped QPMSF devices as measured at full-width-half-maximum (FWHM) are also close to expected values for the given chirp rates.

Figure 1(b) shows the change in the SH conversion efficiency and the bandwidth of the SH spectra as a function of the chirp rate. As expected, there is considerable decrease in the conversion efficiency for increasing chirp rate for devices of identical length. In QPM devices of uniform period, the acceptance bandwidth for efficient conversion decreases with increasing interaction length of the fundamental and second harmonic waves. This inverse relationship places a constraint on the bandwidth of the fundamental source utilisable for efficient SHG. As a consequence long uniform QPM structures are not ideal for frequency doubling of ultra-short pulse sources of broad spectral bandwidth. The ability to precisely control the acceptance bandwidth of the QPM interaction suggests that longer devices can be fabricated and it also enables efficient frequency doubling of high power, ultrashort sources such as femtosecond lasers. The practicalities of this technique increases further for poled silica fibres when compared to bulk silicate or crystalline materials, the advantages of which were listed in the introduction. Current fabrication capabilities allow poling lengths of $1 \mathrm{~m}$ or more in silica fibres. Point-by-point periodic UV erasure of the SON in poled fibres allow tighter control of the boundary tolerances allowing further optimisation of the conversion efficiency and SH spectral profile.

The precise control of the spectral bandwidth of the QPM silica fibres through the introduction of a linear chirp in the SON has been demonstrated for the first time. The spectral profiles of the second harmonic in QPM silica fibres are in good agreement with theoretical predictions. Through this technique, it is now possible to design long QPM structures with bandwidths capable of accommodating ultra-short pulses from high power mode-locked fibre lasers.

[1] V. Pruneri, et al., Greater than $20 \%$ efficient frequency doubling of $1532 \mathrm{~nm}$ pulses in quasi-phase-matched germanosilicate fibres. Optics Letters, 1999. 24(4): p. 208-210.

[2] C. Corbari, et al. All-fibre frequency conversion in long periodically poled silica fibres. in Optical Fiber Conference. 2005. Anaheim, CA, USA: Optical Society of America

[3] M.A. Arbore, et al., Engineerable compression of ultrashort pulses by use of second-harmonic generation in chirped-period-poled lithium niobate. Optics Letters, 1997. 22(17): p. 1341-1343.

[4] T. Suhara and H. Nishihara, Theoretical Analysis of Waveguide Second-Harmonic Generation Phase Matched with Uniform and Chirped Gratings. Journal of Quantum Electronics, 1990. 26(7): p. 1265-1276.

[5] A. Canagasabey, et al. $75 \%$ Enhancement of the Second Order Nonlinearity in Twin-Hole Fibres through Voltage Assisted Cooling during Poling. in The Conference on Lasers and Electro-Optics (CLEO). 2006. Longbeach, CA, USA: Optical Society of America. 